\title{
The EUSO-SPB Instrument
}

\author{
S. Bacholle*1 for the JEM-EUSO Collaboration \\ ${ }^{1}$ Colorado School of Mines \\ E-mail: sbacholle@mines.edu
}

The EUSO-SPB (Extreme Universe Space Observatory on a Super Pressure Balloon) is a balloon borne UV camera. It is dedicated to the observation of fluorescent light produced by UHECR induced air showers from the stratosphere. EUSO-SPB was launched on the 24th of April 2017, 22:50 and lasted for 12 days 12 hours at a nominal altitude of $33.5 \mathrm{~km}$. The main instrument consists of a two Fresnel lens system and a fast UV camera recording images with a time resolution of $2.5 \mu \mathrm{s}$. The camera consists of a pixelized focal surface composed of 36 Multi-Anode photo-multipliers (MAPMT). The optical system includes two Fresnel lenses of $1 \mathrm{~m}$ diameter with a wide field of view. In addition of the main UV camera, EUSO-SPB flew with two auxiliary systems, an IR camera and a SiPM UV camera. In this paper, the different subsystems of the EUSO-SPB instrument will be described: the optical system, the focal surface with its readout electronic, the different subsystems controlling the data acquisition and the auxiliary system.

35th International Cosmic Ray Conference - ICRC2017

10-20 July, 2017

Bexco, Busan, Korea

${ }^{*}$ Speaker. 


\section{Introduction}

EUSO-SPB is a pathfinder for space missions dedicated to the indirect detection of Ultra High Energy Cosmic rays (UHECR). These particles interact with the upper atmosphere and, at an energy of over $10^{18} \mathrm{eV}$, produce Extensive Air Showers (EAS) that can be observed from above.

The principle of the detection of these EAS from space is based of the observation of the fluorescence of nitrogen molecules emitted in the 300-400 $\mathrm{nm}$ spectral range. EUSO-SPB aims at testing the capacity to observe such events from a space observatory.

At a nominal flight altitude of $33 \mathrm{~km}$ and a potential flight duration of several weeks, the long duration stratospheric balloon program of the NASA Columbia Scientific Balloon Facility (CSBF) is a good opportunity to fly a pathfinder to test the EAS detection principles and the instrument in a harsh environment.

EUSO-SPB is flying with a super-pressured balloon allowing to fly for several days, up to several weeks, at a constant altitude even through the day-night transition. It is the successor of a first one-night flight balloon-borne pathfinder, EUSO-Balloon [1], which was launched by the french space agency CNES in 2014 from Timmins, Canada. The primary goal of EUSO-SPB is to detect the first EAS from above, with an energy threshold of $10^{18} \mathrm{eV}$ and a field of view on ground of about $6 \mathrm{~km}$. The different elements making up the EUSO-Balloon payload are described below.

\section{The main instrument}
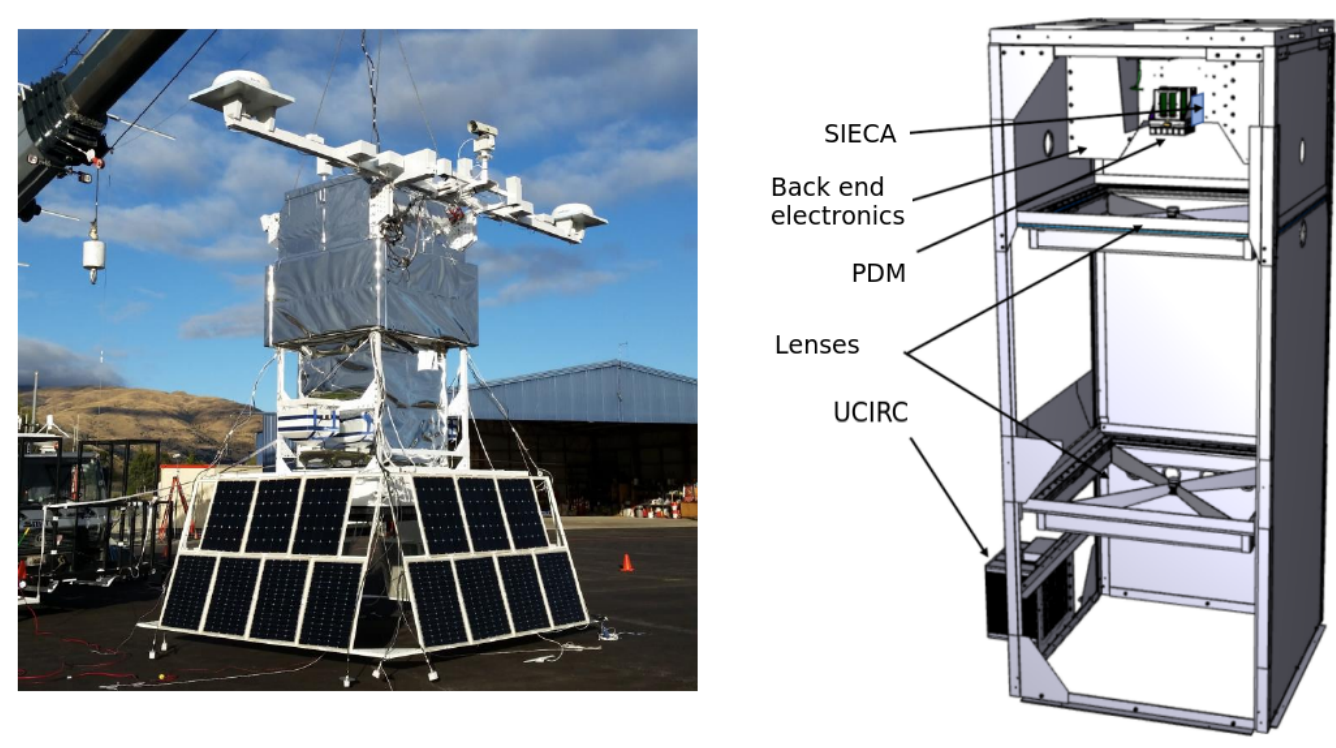

Figure 1: Left: picture of the EUSO-SPB payload during the hang tests. Right: Schematic of the main instrument with the lenses and UCIRC being visible. The solar panel system and the SIP (Support Instrument Package) with the antennas are not shown.

The EUSO-SPB main instrument (see figure 1) is dedicated to the observation of fast UV signal, in particular UHECR induced air showers. The UV camera consists of a $48 \times 48$ pixel focal 
surface (FS). The FS, with the front end electronics and the high voltage power supply, forms the Photo-Detector Module (PDM) which observes the UV light emitted in the field of view provided by two Fresnel lenses.

The back-end electronics, assembled in the Data Processing (DP) mechanical crate, is composed of the subsystems used for the communication between the different parts of the payload, the power supplies, and is at the interface between the instrument and the ground stations. Figure 2 shows a picture of the electronics assembly with a schematic of the electric architecture of the instrument.
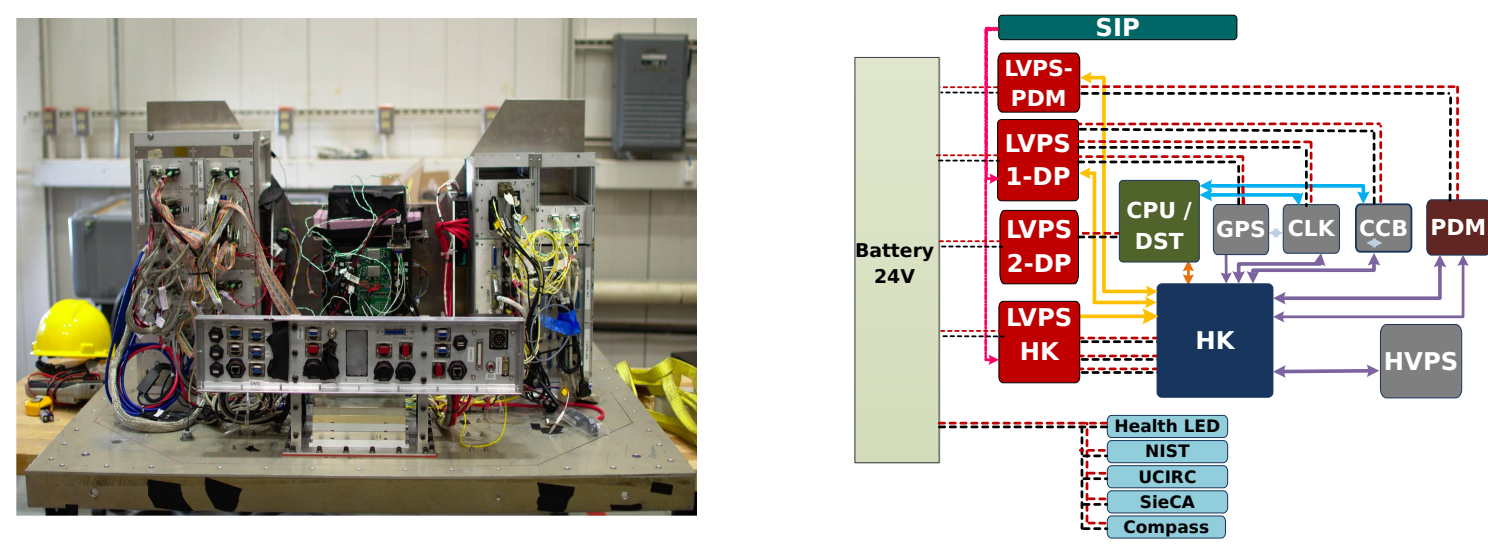

Figure 2: Left: Picture of the electronic assembly with the PDM located between the relay box on the right and the DP crate on the left. Right: electronic architecture of the payload

\subsection{The Photo-detection Module}

The PDM (see figure 3, left) is the main part of the instrument, and is dedicated to the detection, digitalization and pre-processing of the data. The focal surface is composed of $368 \times 8$ MultiAnode Photo-Multipliers (MAPMT), generating a 2304 pixel image. The nominal polarization voltage of the MAPMTs is $+1100 \mathrm{~V}$, with the possibility to change the voltage of the photo-cathode.
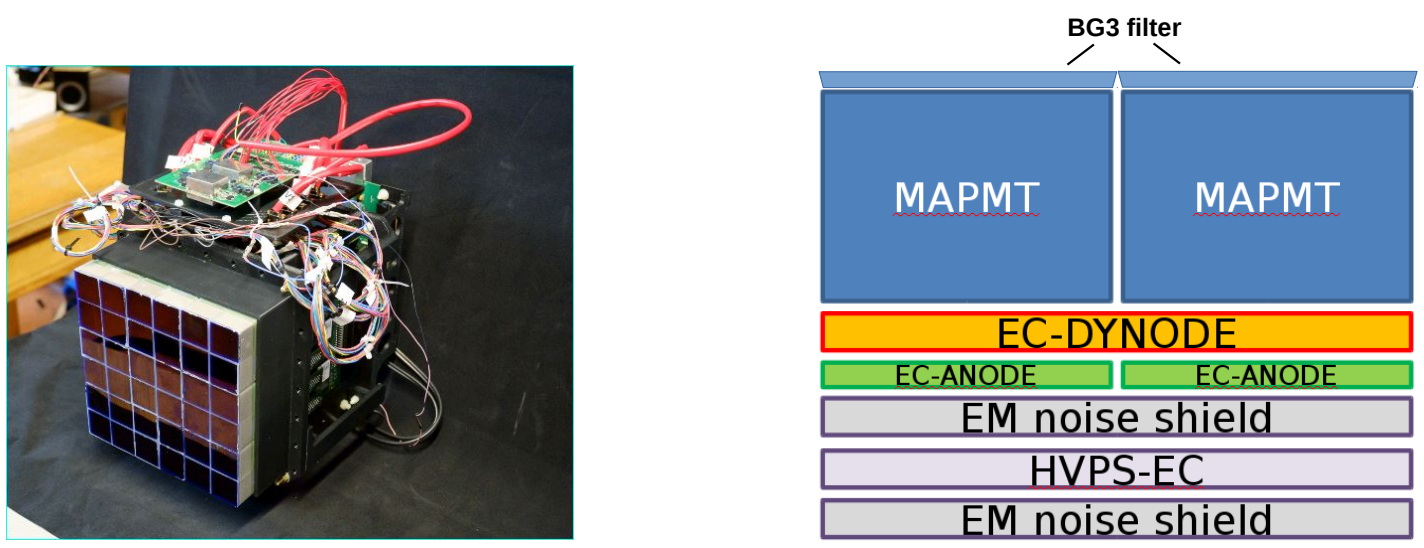

Figure 3: Left: picture of the assembled PDM. One can see the focal surface made of 36 MAPTM and their BG3 filters, and the HVPS board on the top. Right: Diagram showing the different element of the EC. 
The Elementary Cell The focal surface is segmented in groups of $2 \times 2$ MAPMTs called Elementary Cell (EC). Each EC is a compact assembly containing one HVPS generator board powering the four MAPMTs and the EC-anode boards bringing the analog signal from the MAPMT to the ASIC. Each EC is "potted" in a gelatinous compound to prevent discharge between the different components at the low pressure of EUSO-SPB altitude. A schematic of the EC is shown on figure 3 on the right.

The MAPMTs are operating in photon counting mode. When a photon hits the photo-cathode, an electron (called photo-electron, p.e.) is produced with a probability depending on the quantum efficiency of the cathode. After being amplified with a gain of the order of $10^{6}$ in the electronmultiplication stage of the MAPMT, the anode pulse at the output of the MAPMT can be read by the read-out electronics. When the photon detection rate is low enough each pulse can be separated and the output signal of the PDM is equal to the number of p.e. produced. The total PMT efficiency is the result of the quantum efficiency with which a p.e. is generated at the photo-cathode, and the collection efficiency which characterizes the number of p.e. reaching the first dynode and depends on the voltage difference between the cathode and the first dynode of the MAPMT.

The HVPS system The HVPS system [2], is composed of one HVPS board per EC which hosts the high voltage generators, and a HVPS control board. The generators are Cockcroft-Walton voltage multipliers which provide the voltage to the dynodes and anodes of each MAPMTs of the EC. The HVPS board is providing insulation and communication between the HVPS generators and the rest of the instrument, and commands the HV level.

EC-ASIC boards The analog signal of each MAPMT is sent to the EC-ASIC boards. Each of them are composed of 6 SPACIROC3 ASICs developed by Omega (CNRS, France). The ana$\log$ signal at the output of each MAPMT is processed by the corresponding ASIC. The signal is first amplified, before being transformed into trigger pulses and digitalized by an ADC. The SPACIROC3 ASIC allows a double pulse separation at $100 \mathrm{MHz}$ with a power consumption of around $0.7 \mathrm{~mW} / \mathrm{channel}$, which fulfills the requirements for a space base mission $(1 \mathrm{~mW} /$ channel $)$.

PDM board The FPGA is hosted in the PDM board. Its main role is to receive the digital data from all the ASICs, before sending the complete packet to the disk. In the FPGA, the data is being processed by the first level trigger [3] in order to discard background noise.

The FPGA is also managing the switching algorithm. This program is dedicated to switching down the voltage of the MAPMT photo-cathode of each EC when the light reaches a previously set threshold over an entire EC to prevent damaging the MAPMT. The cathode voltage can be lowered to either the voltage of the first dynode, or to $0 \mathrm{~V}$ to completely nullify the collection efficiency of the MAPMT. Decreasing the voltage of the photo-cathode only decreases the collection efficiency of the MAPMT. The gain of the whole electro-multiplication chain remains the same, so the MAPMT is still operating in the photon-counting mode.

\subsection{The Data Processing unit and subsystems}

The back end electronic is composed of the CPU-disc assembly, the GPS, clock board and Control Cluster Board (CCB), all located in the same mechanical crate with the LVPS modules and the House Keeping (HK) module. The primary role of the CCB is to manage several PDMs 
and to host the 2nd level trigger. Although EUSO-SPB did not use any of these functions, a good integration of the CCB is an important step in making a proof of concept for bigger space telescopes using several PDMs due to its importance in the communication and data flow during the operation of the instrument.

The HK had the double role of receiving ON/OFF and operational commands from the ground operation centers to the instrument, as well as sending back status and health data from the different subsystems. The Data Processing unit and the software are described in more details in [4]

The different low voltage power supplies are also incorporated in the DP crate. They provide low voltage to the subsystems from the batteries output and each of them have their own ON/OFF through hard relays controlled from the ground by the SIP provided by CSBF.

\subsection{Ground operation}

The data was transmitted through the two IRIDIUM PILOT antennas to the Ground Support Equipment at the CSBF facility in Palestine, Texas. From there, the data was sent to a server based at the Colorado School of Mines. After the monitoring of the instrument from Wanaka with the line of sight link, the flight was followed from several command centers: Colorado School of Mines (USA), Riken (Japan), the University of Tubingen (Germany), IRAP (France), the University of Torino and University of Naples (Italy).

In addition to the status of the instrument (ON/OFF, voltages, temperature, etc) a small portion of the photo-detection data was downloaded as soon as it was acquired to check the wellness of the focal surface and front end electronic. An UV LED was set on the mechanical structure of the first lens and was directly illuminating the camera with a double pulse at a regular intervals.

In order to support the decision to turn ON or OFF the MAPMTs, two photodiodes precalibrated by the National Institute of Standards and Technology (NIST) were attached to the side of the PDM and constantly measuring light.

\subsection{The optics}

The optical system of EUSO-SPB is composed of two Fresnel lenses of 1 meter diameter, $110 \mathrm{~cm}$ apart. The lenses, made in PMMA, were manufactured in RIKEN (Japan). The performances of the optics were evaluated at Colorado School of Mines with an optical bench set up to measure the Point Spread Function (PSF) and the optical efficiency of the system. The field of view was measured in the Telescope Array site in Utah with laser pulses and by observing the night sky and the inclination of bright stars. The measurements gave a field of view of $11.10 \pm 0.15^{\circ}$ and a PSF of $10 \mathrm{~mm}$ with a throughput of $30 \%$. These measurements are described in [5].

\subsection{The solar power system}

The payload was powered by solar panels which charged a battery pack to provide power during the night. The solar power system is composed of $27 " \times 31$ " SunCat panels disposed all around the payload on a crinoline. The battery pack consists of five pairs of $12 \mathrm{~V}$ Odyssey batteries connected in series to give a nominal voltage of $24 \mathrm{~V}$. Both the batteries and solar panels were flight proven by CSBF. Each panel is able to produce a nominal power of 100 Watts when the Sun was normally incident on the panels. The power system was connected to Morning star Sunsaver MPPT controllers to control the charging cycle of the batteries. 
The peak power requirements for the payload was $150 \mathrm{~W}$ with an average of $75 \mathrm{~W}$ in normal conditions during the night. So with each side of the crinoline having three panels, the power produced by the solar panel system exceeds the power required to run the instrument.

\subsection{The gondola}

The innermost structure was formed by the gondola used in the recovered EUSO-Balloon experiment. The gondola is made of $10 \mathrm{~mm}$ thick Fibrelam®panel and is composed of two parts, the optical box and instrument booth. These two parts are independent, allowing to completely integrate the electronic system before assembling the whole instrument.

The gondola is integrated in an exoskeleton which supports the rest of the payload: the SIP, the antenna boom and the solar panel crinoline. The requirement of the mechanical structure was to support ten times the weight of the payload applied vertically to the suspension point, and five times the weight applied horizontally to the suspension points and the attached equipment. A rolling cart was made to perform the integration of the gondola in the exoskeleton and the manipulation of the payload in and out the hanger in Wanaka, as is shown on figure 4. Both the exoskeleton and the rolling cart were made at Colorado School of Mines.
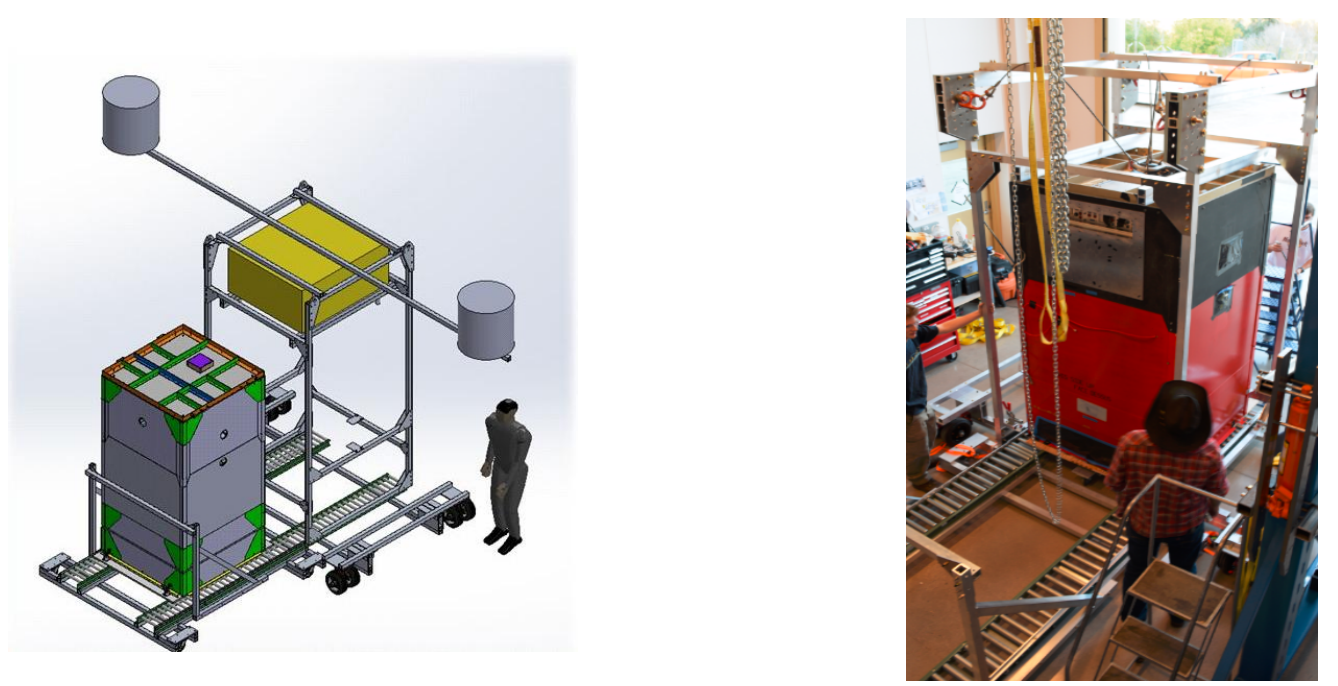

Figure 4: Left: Schematic of the gondola on the cart before integration in the exoskeleton. Right: picture of the gondola in the exoskeleton

\section{The auxiliary systems}

In addition to the PDM, EUSO-SPB was fitted with several auxiliary instruments used in combination with the main camera. UCIRC is a infrared camera and provides observation of clouds and atmospheric events in the field of view. SIECA is a prototype of a silicon PM camera; its role is to assess the capability of SiPMs in UHECR air shower detector. 


\subsection{UCIRC}

The knowledge of the atmospheric conditions in the field of view of the instrument is important to determine the exposure of EUSO-SPB. As UHECR air showers can partially develop under clouds, the exposure depend on their optical thickness and altitude.

In order to measure these parameters, infrared images are taken by the University of Chicago IR Camera (UCIRC) during each night. The instrument is composed of two IR cameras fitted with different bandpass filters. One camera is using a 11.5-12.9 $\mu \mathrm{m}$ and the other is sensitive between 9.6 and $11.6 \mu \mathrm{m}$. Its large field of view ( $32 \times 24$ degrees), larger than the UV camera field of view, gives the possibility to measure the cloud coverage in the field of view of the PDM between pictures.

UCIRC had its dedicated power supply, and was controlled independently than the PDM. The pictures, taken every 16 seconds, were stored in the main disk and downloaded in parallel to the PDM images. UCIRC is described in [6] and a picture of the instrument is shown on figure 5 on the left.

\subsection{SIECA}

Silicon Photo-Multipliers can theoretically give the same low light sensitivity as the high voltage PMTs. Contrary to PMTs, SiPM operate at a much lower voltage. They also benefit from a small form factor allowing an easier integration, as one can see on figure 5 on the right.

The Silicon photomultiplier Elementary Cell Add-on (SiECA) camera's purpose is to test this technology in space conditions in the context of indirect detection of UHECRs. The focal surface is composed of one Elementary Cell made up of 4 SiPMs containing $8 \times 8$ pixels behind BG3 filters. The surface of the EC is thus similar to the surface of a PDM EC. SIECA has its dedicated $5 \mathrm{~V} / 2 \mathrm{~A}$ power supply which is enough to provide all the required voltage for the camera, including the operational voltage of the SiPMs and the front end electronic composed of an FPGA and four ASICs.

SIECA was attached to the PDM with the EC located on the edge of one of the ECs of the PDM. The acquisition was triggered by the PDM acquisition with a pulse sent by the CPU with a varying delay depending of the CPU clock. More information can be found about SIECA in [7].
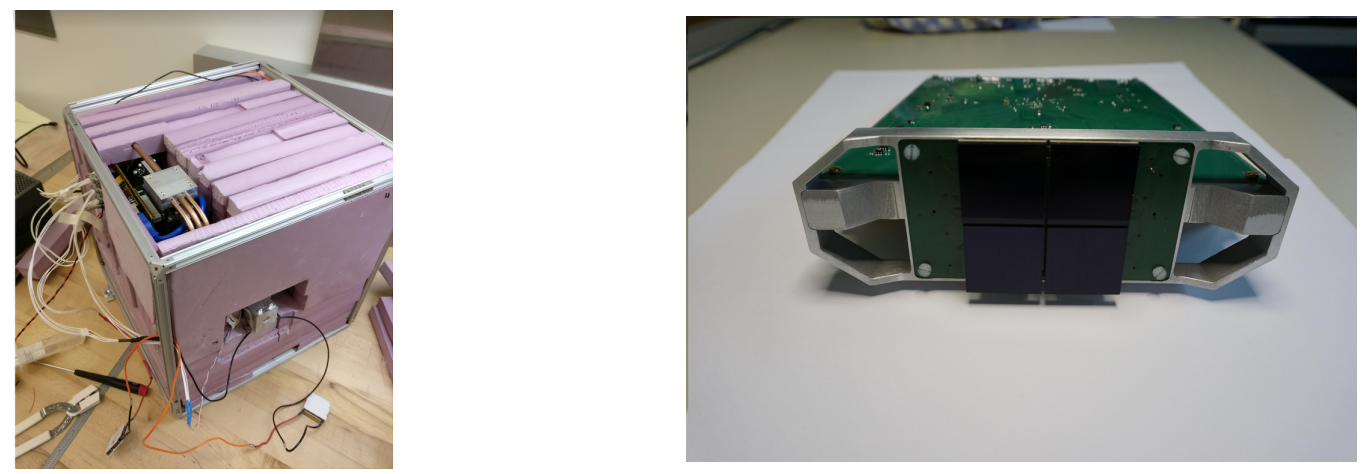

Figure 5: Left: the UCIRC camera. One can see the passive cooling system on the top, and one of the Peltier cooler on the front. Right: the SIECA camera, with two of the four SiPM, two of the ASICs and the DAQ board. 


\section{Thermal model}

At an altitude of $32 \mathrm{~km}$, the payload can be subject to a large range of temperature with the day/night cycle. Moreover, at low pressure, the heat regulation by convection is very small and most of the temperature flow is done by radiation and conduction. The thermal model was showing temperatures at the gondola reaching $-20^{\circ} \mathrm{C}$ and the temperature of the PDM fluctuating between $0^{\circ} \mathrm{C}$ and $5^{\circ} \mathrm{C}$. The most critical elements were the HVPS generator in the EC which are rated for temperatures above $0^{\circ} \mathrm{C}$. To prevent malfunction in case of lower than expected temperatures, Kapton flat heaters controlled by a thermostat were affixed on the four sides of the PDM.

\section{Conclusion}

The EUSO-SPB instrument flew for 12 days after a successful integration and tests, first at the CSBF center in Palestine, Utah, and in Wanaka in New-Zealand. After a commissioning period of two nights, the UV telescope was recording light in nominal instrumental conditions and all the subsystems could work together as expected. Some improvements over the first EUSO-Balloon flight in August 2014 were successfully tested, in particular the integration of the first level trigger, a new architecture of the ECs hosting the HVPS generators, and a new version of the ASICs.

\section{Acknowledgment:}

This work was partially supported by NASA grants NNX13AH55G, NNX13AH53G, the French Space Agency (CNES), the Italian Space Agency through the ASI INFN agreement n. 2017-8-H.0, the Basic Science Interdisciplinary Research Projects of RIKEN and JSPS KAKENHI Grant (22340063, 23340081, and 24244042), the Deutsches Zentrum für Luft und Raumfahrt, and the 'Helmholtz Alliance for Astroparticle Physics HAP' funded by the Initiative and Networking Fund of the Helmholtz Association (Germany). We also acknowledge the NASA Balloon Program Office and the Columbia Scientific Balloon facility for extensive support, the Telescope Array Collaboration for the use of facilities in Utah.

\section{References}

[1] P. von Ballmoos, for the JEM-EUSO Collaboration, The EUSO-Balloon pathfinder in proceedings of the 34th ICRC (ICRC2015) 0725, 2015.

[2] J.Szabelski, Z.Plebaniak, J.Karczmarczyk, W.Marszal, P.Prat, P.Gorodetzky, G.Prevot, JEM-EUSO Collaboration, HVPS system for *-EUSO detectors. in Proceedings of the 35th ICRC (2017)

[3] J.Bayer, M.Bertaina, M.Mignone, H.Miyamoto, J.Eser, JEM-EUSO Collaboration, The trigger logic of EUSO-SPB and its performance in Proceedings of the 35th ICRC (2017)

[4] G.Osteria, V.Scotti, F.Perfetto, F.Cafagna, C. Fornaro, JEM-EUSO Collaboration, The Data Processor System of EUSO-SPB . in Proceedings of the 35th ICRC(2017)

[5] J. Eser et al., JEM-EUSO Collaboration, Preflight calibration and testing of EUSO-SPB in the lab and the desert in Proceedings of the 35th ICRC (2017).

[6] L. Allen, M. Rezazadeh, S. Meyer and A. V. Olinto, JEM-EUSO Collaboration, UCIRC: Infrared Cloud Monitor for EUSO-SPB in Proceeding of the 35th ICRC (2017).

[7] W. Painter et al., JEM-EUSO Collaboration, Development of Silicon Photomultiplier Prototype for UHECR Fluorescence Detection from Space in Proceedings of the 35th ICRC. 